\title{
La caracterización de la enseñanza como conjunto de técnicas para intervenir en los aprendizajes: un análisis crítico
}

The characterization of teaching as a set of techniques for intervention in learning: a critical analysis

Luis Ernesto Behares* Universidad de la República

Resumo Este trabajo indaga las condiciones de producción del traslado de la atención investigativa y operativa desde las figuras de la enseñanza a las del aprendizaje. La enseñanza, entendida como el campo de la conservación del conocimiento históricamente constituido, en su encuentro con las dinámicas de la inestabilidad y la contingencia que llamamos saber, incluye el aprendizaje, pero no se agota en él. El aprendizaje es la respuesta dada por los individuos en sus procesos adaptativos y de construcción de sus herramientas intelectuales y ha requerido para su validación en el campo de la enseñanza los aportes de la psicología.

La proclamación de la centralidad de los procesos del aprendizaje durante el siglo XX en el campo pedagógico es una transformación teórica e ideológica capital, que buscamos explorar con minuciosidad desde la perspectiva dialécticamente opuesta, conocida como teoría del acontecimiento de enseñanza.

PALAVRAS-CHAVE: Enseñanza; Aprendizaje; Teoría de la acción; Teoría del acontecimiento.

Abstract This paper investigates the conditions of production in which research and operational attention changed from a focus on teaching to a focus on learning. Teaching, understood as the field of historically constituted knowledge, which encounters the instability and contingency we name cognizance, includes learning, but it also goes beyond it. Learning is the individuals' response in their processes of adaptation and construction of their intellectual tools, and it has required the contribution of psychology for its validation in the field of teaching.

The claim of the centrality of learning processes during the 20th century in the field of pedagogy is a key ideological and theoretical transformation. We intend to explore it thoroughly from the dialectically opposed perspective of the theory known as event of teaching.

KEYWORDS: Teaching; Learning; Theory of action; Theory of event of teaching. 


\section{Propósito}

Este artículo ${ }^{1}$ intenta recoger y analizar el traslado de las cuestiones principales del campo teórico de la enseñanza, inscriptas también como propias de la didáctica, al campo del aprendizaje y, por ende, parte de considerar las construcciones pedagógicas, psicológicas e ideológicas que sirvieron como catalizadores de ese traslado. Este proceso se operó en Estados Unidos, Europa y nuestros países latinoamericanos durante el siglo XX, aunque estemos acostumbrados a él y lo tengamos fuertemente naturalizado.

En el marco de las concepciones presididas por la escuela nueva, los pragmatismos y la fuerte presencia de las psicologías empiristas en el campo educativo, el siglo $\mathrm{XX}$ ha acuñado una definición de enseñanza como aquellas técnicas que se construyen para intervenir en los aprendizajes. Es esa construcción la que abordamos aquí.

Una larga serie de autores han tratado esta cuestión desde muy distintos puntos de vista, entre los cuales señalaremos los que más se relacionan puntualmente con nuestro análisis: Coll (1988), Chevallard (1998), Lajonquière (1999), Saviani (2000), Del Río y Álvarez (2010), Behares y Voltolini (2011), Behares (2014) y Fernández Caraballo (2014). En cualquiera de esos textos, se reconocen los parámetros de la construcción teórica que nos interesa y muestran que ella tiene innegables efectos sobre la comprensión de la enseñanza, tanto en su dimensión heurística como en su dimensión de diseños operativos y de políticas escolares.

Es fácil constatar en la extensa bibliografía al respecto que el nuevo concepto que hizo centro en las teorizaciones es el de aprendizaje. En los marcos de referencia más o menos filosóficos y pedagógicos con que se promovió la centralidad del educando en el proceso educativo, se situó a sus procesos mentales y a su interioridad de individuo en el centro de las atenciones.

\section{La centralidad de los aprendizajes}

La palabra "aprendizaje" es lo suficientemente ambigua en cualquiera de las lenguas (aprendizaje, learning, Lehrzeit, etc.) y además ha coexistido con otros términos que la circundan según las tradiciones teóricas de la propia psicología (desarrollo, adquisición, construcción, competencia, etc.). Aún así fue presentada con fuerza argumentativa por los conductismos de inicios del siglo en sus diferentes versiones. Se trató, en cada una de las modalidades que los conductismos forjaron, de proponer una teoría del procesamiento interno-externo de los individuos en relación con su experiencia, a veces representada como estrictamente orgánica, otras como orgánico-social y otras como intersubjetiva, con ciertos visos de "mentalismo", pero estableciendo siempre un programa positivo, observable y científicamente riguroso.

El texto fundador de Thorndike (1903) determinó la construcción conductista del objeto propio de la psicología educacional. El aprendizaje se constituye como la materia de la que tratan las acciones educativas y de enseñanza. Estas acciones deben concebirse como formas de intervención, con uno u otro formato práctico, en 
las dinámicas de la acción de los aprendientes. Desde un punto de vista teórico y epistémico, la teoría del aprendizaje pasa a presidir la teorización sobre la enseñanza. Otros modelos posteriores se inscriben, al modo positivista crítico, en esta tarea. Es el caso de los conductismos posteriores más influyentes en los Estados Unidos (e. g. BRUNER, 1966; AUSUBEL, 1968; SKINNER, 1968).

Otro tanto puede decirse de las trasformaciones ocurridas en la tradición europea, donde las visiones pedagógicas pasaron a estar representadas por modelos psicológicos de comprensión derivados principalmente de la obra de Piaget, aunque también de otros autores. El fundador de la epistemología genética (PIAGET, 1950) sirvió con su instigante producción sobre los procesos de construcción cognitiva de los niños al desarrollo de una corriente psicológica acrecida fundamentalmente en los ámbitos de la enseñanza y la educación. Esta corriente, con sus vertientes constructivistas, se pliega a la tarea de pensar una enseñanza escolar que "acompañe" al proceso de construcción infantil de estructuras y de contenidos cognoscitivos y también una psicopedagogía esencialmente terapéutica (Cfr. VUYK, 1984; LAJONQUIÈRE, 1999, p. 55 y ss.).

Otras tradiciones no dejan de inscribirse, aunque es justo anotar que a veces con cierta dificultad, en esta determinación de centralidad de los aprendizajes. Así, por ejemplo, el trabajo de Vigotsky anterior a 1934 en la Unión Soviética pasó a difundirse desde los Estados Unidos a partir de 1962 como una suerte de conductismo social crítico. Los intentos marxistas de Vigotsky en textos como el de su primera conferencia de 1924 (VIGOTSKY, 1991) o más avanzados en su producción en 1931 (VIGOTSKY, 1995), relativamente complejos y contradictorios al relacionar filogénesis y ontogénesis, así como por su fina distinción entre aprendizaje y desarrollo, fueron reinterpretados desde la teoría de la acción estadounidense, por autores de la influencia de Wertsch (1985) como un proceso conductual-mental de tipo funcionalista, interpretación también difundida en otros territorios académicos, por ejemplo en España, (.por ejemplo, DEL RÍO; ÁLVAREZ, 2010).

Sobre las bases de alguno de estos modelos, y de otros semejantes, la importación de lo psicológico a las teorías de la enseñanza se operó para construir un aparato que permitiera entender la relación enseñanza-aprendizaje en términos positivos, estables, planificables, controlables y evaluables. La tecno-teoría de la enseñanza característica de gran parte del siglo XX se hizo posible gracias a la jerarquización epistémica de esa relación. Desde la década de 1930, en el campo académico de la enseñanza decayó todo y cualquier interés conspicuo sobre las cuestiones "teórico-explicativas", y pasaron a preponderar los énfasis investigativos sobre los aspectos "tecnológico-instrumentales" y "técnico prácticos" (COLL, 1988, p. 178-189). Como es obvio, las cuestiones teórico-explicativas eran provistas por modelos importados de la psicología y, a posteriori, acomodadas y reinterpretadas como instrumentos explicativos del accionar tecno-didáctico. Este carácter interdisciplinario aplicacionista determinó el traslado de la teoría de la enseñanza a la psicología de la educación. 
Dos factores de diferente origen aunados hicieron posible ese traslado:

a) la episteme psicológica, que como conditio sine qua non sustenta la presencia central del in-dividuo (el ego) en todo y cualquier proceso, como centro objetivo del accionar, sea este "mental", "psíquico", "social” u "orgánico”, y

b) la tendencia romántica de las corrientes pedagógicas del siglo XX (sobre todo las calificadas como "escuelanovistas") a inspirarse en la "comunicación con los niños” como justificador de las políticas de enseñanza.

Es posible sostener, como lo ha hecho Behares (2014), que la enseñanza pasa a estar regida por la centralidad de los procesos iinteriores-exteriores de los individuos que son su objetivo: los niños. Pero más allá de una cuestión cronológica o de naturaleza biológica ligada al desarrollo, se trata de una composición más general, en la cual se postula al individuo o ego como único agente, o agente central patrocinador de todos los procesos que atañen al mundo humano en todos sus componentes.

Es evidente que el ego patrocinante dió lugar a una noción de enseñanza muy diferente a la que se tenía a inicios del siglo XX, que para sostenerse en sus propios términos requirió reestructuras teóricas radicales y conceptos nuevos muy bien definidos. De hecho, esta noción supone la representación de la relación sujeto-conocimiento como estrictamente apropiativa, ya que el aprendizaje que es su centro epistémico remite invariablemente al adaptacionismo conductista o al productivismo cognitivista. Pero, y al mismo tiempo, la caracterización egoica de la enseñanza-aprendizaje ha requerido, con el tiempo, la incorporación de modelos representativos provenientes de la psicología interaccionista e intersujetivista, provistos desde los años de 1970 principalmente por el eclecticismo bruneriano de esos años (ver, principalmente, BRUNER, 1984).

Según estos modelos, la enseñanza debe ser pensada como un dispositivo de aula centrado en la interacción entre profesor y alumno, en interpretaciones más o menos formales y tecnicistas (en las cuales los individuos allí presentes realizan una acción unos sobre otros), o en versiones más reguladas por las circunstancias sociales y personales, mediante la "negociación" en la cual se acomodan los unos a los otros. El psicologismo de los años de 1980 hizo presente la interacción entre las figuras egoicas profesor-alumno como la esencia misma de lo didáctico. Es así que se la representa en términos de la trasparencia comunicativa, en la cual juega un papel determinante la posibilidad de concebir a los sujetos como egoicos e individuales.

El interaccionismo tuvo un efecto interesante: llamar la atención del fenómeno de aula como un objeto de investigación en sí mismo, con cierta distancia, no siempre tan marcada, es verdad, entre el fenómeno en sí y las preceptivas didácticas, en la medida en que las constantes y variables que se presentan en ese fenómeno superan a cualquier modelo preceptivo. En términos generales, esa apropiación epistémica se hizo en base a la teoría de la acción: el aula es un acto que ocurre entre el niño (o un grupo de éstos) y su maestro. 
La teoría de la acción tuvo como uno de sus principales formuladores en los Estados Unidos a Parsons, que en uno de sus primeros libros, publicado en 1937 (PARSONS, 1968) define el "acto-unidad" como la acción producida por un agente o actor, limitada por la situación y los otros participantes en ella. De esta forma, el actor es el agente de su acción, pero ésta está también dominada por los factores que el actor no puede controlar, a causa de que hay una "suma de voluntades" inter-actuando en un contexto de orientación social predeterminado.

La acción, como medio para cumplir un fin, se afianza en la voluntad (lo que el actor quiere hacer con ella) y en la percepción de las prefiguraciones sociales que pueden, o no, llevarlo a su meta. La acción de otros, que puede o no coincidir con la suya, también se inscribe en estos límites prefigurados. En textos posteriores (PARSONS; SHILDS, 1951) se acentúa, vía la apelación a las teorías de la comunicación, dos caracteres: los juegos interaccionales transparentes de voluntades y las prefiguraciones sociales estructurales observables y caracterizables.

Tolman (1932) incorporó a la tradición estadounidense el principio de que la conducta (definida esencialmente como acción), está formada por respuestas que definen sus significados en la acción: una acción o una conducta dadas tienen que ser identificadas y definidas sólo según las formas en que tienden a manipular o reordenar los objetos culturales, sociales o físicos que se hallan en relación con un actor determinado.

Tanto Parsons como Tolman cimentaron la concepción que enfatiza los actos (entre los cuales los procesos mentales) como objetos de estudio de la psicología o la sociología, mediante el punto de vista funcional: la mente y las relaciones sociales deben ser estudiadas en función de su utilidad para las necesidades de los organismos, la adaptación de éstos a su medio y las expectativas sociales establecidas, fieles a los esquemas ideológicos del individualismo filosófico y del liberalismo político inherentes al pensamiento estadounidense.

Allport (1968), sin mucha distancia con lo que acabamos de exponer, señaló que el individuo constituye su personalidad en la acción, y puso el énfasis en el carácter único del individuo, una vez que éste es concebido como un organismo inherentemente activo, y no solamente reactivo. La individualidad es, en su concepto, la resultante cualitativa del sistema de interacciones que cada sujeto logra sostener, o, en otras palabras, las diferencias cualitativas que cada sujeto presenta en relación a los otros y al patrón de medida general que pueda establecerse. Esta forma de definir la individualidad apunta al estudio global de su estructura, la personalidad, que pasa a ser definida como la organización dinámica en el interior del sujeto de los sistemas psicofísicos que determinan su conducta y su pensamiento característicos. Como unidad egoica indivisible, el sujeto que será luego caracterizado como "bio-psico-social", lo es en tanto resultado directo de sus acciones.

La presentación del sujeto como producto de su acción en el marco de referencia "bio-psico-social" es constitutiva del modo interaccionista de situar el aula como objeto de indagación. En este modo, encausar los estudios sobre la enseñanza, y 
aunque muchas veces se pase por alto que de eso se trata, hay una reducción considerable del objeto a las prácticas activas de enseñantes y enseñados como personas independientes en ocasión de un objetivo común o socialmente prefigurado. Los llamados estudios de "interacción en el aula" descansan, a pesar de la enorme variedad de enfoques interpretativos, en esa constante teórica. Entender el aula como comunicación, o como conjunto de actos de interacción empírica entre los participantes en ella, implica reducir la teoría de la enseñanza a la dimensión inmediata de la acción trasparente.

Las corrientes de análisis denominadas "interaccionistas", "conversacionales" o "discursivas", generadas en los intersticios entre la lingüística, la antropología, la sociología y la psicología, no tenían la intención ni las posibilidades de inscribirse en las teorizaciones sobre la enseñanza, pero produjeron instrumentalidades que se insertaron naturalmente en ella. Las conceptualidades y modelos de abordaje de autores como Bourdieu (1982), Gumperz (1982), Stubbs (1983), Coulthard (1985), Cazden (1991), Schiffrin (1996), Kerbrat-Orecchioni (2001) y Fairclough (2003), entre muchos otros de diferentes orígenes y con programas de investigación bastante disímiles, resultaron amalgamados en estudios puntuales sobre aspectos de la interacción en las aulas de todos los niveles de la enseñanza, en el marco de referencia de una marcada "interdisciplinariedad" de tipo "complementario" (término que usamos en el sentido de HENRY, 1992, pp. 113-153).

\section{Enseñanza, interacción y saber}

Pensar la enseñanza como un acto de interacción entre personas empíricas, aun cuando se establezcan restricciones estructurales para esas interacciones, supone la reconsideración de la enseñanza en sí misma, como composición teórica. En principio, esta composición teórica descansa sobre dos postulados necesarios:

a) la enseñanza consiste en la interacción misma, concebida como acomodación de los interactuantes entre sí, en sus lugares egoicos de acción, con transparencia relativa y con procesos objetivizables, y

b) la enseñanza es un acto psico-bio-social, posibilitado por instrumentos de comunicación, como el lenguaje, en el que transitan "contenidos" de conocimiento, creencias, representaciones de experiencias personales y otros productos de la acción.

Caracterizada de esta manera, la enseñanza es, toda ella, del orden de los actos. El saber/conocimiento se concibe como exterior a ella y se reduce a un conjunto de posibles contenidos, que no son los determinantes principales.

El saber/conocimiento, empero, se justifica dialécticamente por la historicidad continua del pensamiento humano y por las discontinuidades que le imponen la ciencias, no menos históricas; presenta una condición básica de anterioridad a la experiencia y a las dinámicas pragmáticas (es decir, de los actos individuales), que es del orden de un funcionamiento impersonal e histórico. Este carácter de herencia epistémica ha sido siempre sustentado por la epistemología y la historia de la ciencia, pero en general se deja de lado en el campo de la enseñanza y de la pedagogía. En el campo 
normalista y de formación de docentes para la enseñanza básica existe una distinción explicita o contenida en los curricula entre dos tipos de didáctica, la general y la específica, que nos permitiría ver cómo se tratan las cuestiones del conocimiento-saber en él.

La didáctica general se construye en el generalismo y la adaptación escolar del conocimiento, o sea, la reconstrucción global y continua del conocimiento que rige la enseñanza como marco de referencia ${ }^{2}$. Es subsidiaria de la llamada "enseñanza educativa”, dirigida a los niños con intencionalidad pedagógica, ya que el proyecto pedagógico de la modernidad incluyó una variedad de la enseñanza dirigida a los niños de acuerdo a las posibilidades atribuidas a éstos por sus educadores.

La expresión "enseñanza educativa" (erziehende Unterricht) apareció ya a principio del siglo XIX, probablemente en los textos de Herbart ${ }^{3}$, quien la utilizó para referirse a la centralidad de la enseñanza en el proceso pedagógico, que denominaba educación por la enseñanza (o instrucción, en algunas traducciones). Posteriormente, algunos de sus discípulos y difusores, que estuvieron implicados en la construcción de sistemas didácticos y de formación de enseñantes en Alemania y en los Estados Unidos, la utilizaron desde 1850 con el sentido de enseñanza integrada a la atención de los niños y que debía darse en el contexto de la enseñanza primaria. La enseñanza educativa es un elemento caracterizador de la enseñanza escolar y en la tradición normalista desde entonces, como lo consignan los diversos autores clásicos que han estudiado este proceso (DE GARMO, 1889; SCHWENK, 1963; ROERICH, 1884; MAUXION, 1901; COMPAYRÉ, 1904) ${ }^{4}$ y se mantiene inalterable hasta nuestros días.

Las didácticas especificas se constituyen en relación a los campos disciplinarios de conocimiento instituidos fuera de la educación. De hecho, las didácticas específicas han sido el modelo característico de la enseñanza superior, mientras que las didácticas generales lo han sido de la enseñanza primaria. Las tendencias "disciplinaristas" de ciertos modelos de enseñanza secundaria o media se corresponden con las didácticas específicas, si bien las tendencias pedagógicas las retrotraen usualmente a la didáctica general ${ }^{5}$.

Es verdad que en estos esfuerzos de caracterización de la enseñanza el saber/ conocimiento está concebido como una propiedad más de la mente, ya que a ella se le atribuye una condición procesal de tipo "cognitivo" o "cognoscitivo" y según los parámetros teóricos que para definir esos procesamientos se han presentado en diferentes versiones, siempre provenientes de alguna psicología. Si observamos la bibliografía muy extensa generada por esta corriente de interpretación, nos encontramos que la discusión sobre la mejor o la peor enseñanza se da siempre en términos de adecuación psicológica, casi podríamos decir que son, en su esencia, discusiones psicológicas. De este modo, la nueva teoría de la enseñanza deriva todo el abordaje de su relación con el saber/conocimiento de la dimensión estrictamente psicológica, unida mejor a los aprendizajes que a cualquier otro componente, toda vez que se sustenta en una inexorable hipóstasis del guión $(x-y)$ en la secuencia enseñanza-aprendizaje.

Es justo señalar que los pragmatismos y las versiones más enjundiosamente tecnicistas fueron objeto, ya desde los años de 1950, de profundas críticas pedagógicas, sin olvidar las discusiones acerca de sus alcances dentro de la misma psicología de la 
educación. Esas discusiones, a ambos lados del Atlántico, revelan innumerables inconsistencias inherentes a estas corrientes, aunque también las críticas provenientes de lo que Bowen (1979, p. 313 ss.) llamó "reacciones conservadoras", sobre todo en Europa a partir de los años de 1930. En los Estados Unidos, estas críticas estuvieron ligadas a los diferentes problemas de la educación en ese país, a raíz de lo cual los tecnicismos insistentes fueron severamente cuestionados. En América Latina debe destacarse una reflexión académica y pedagógica muy desarrollada, que, entre otros muchos ejemplos, llevó a Saviani $(1985,2000)$ a al entredicho, desde una lectura pedagógica, de la "escuela nueva" y sus derivados.

En términos de la teoría de la enseñanza, la versión pragmatista y psicologista que hemos analizado conserva su hegemonía en sus márgenes y límites: es en ella que las investigaciones sobre enseñanza y aprendizaje se desarrollan mayoritariamente. Sin embargo, la producción teórica sobre la enseñanza no se ha limitado en los últimos treinta años a ese formato, ya que las "cuestiones viejas" que ella opacó, continúan siendo válidas en nuestra modernidad tardía y nos obligan a poner en duda los constructos imperativos de la modernidad.

La teoría moderna de la enseñanza ha perdido o ha hecho evanescentes los elementos necesarios para dar cuenta de lo que en la teoría antigua era de una obviedad evidente: las relaciones entre el saber y el conocimiento y la condición de palabra característica de la enseñanza. Si esa evaluación es aceptable, se requeriría un "retorno" a la teoría antigua, no para eliminar ingenuamente los efectos de la modernidad, sino para iluminarlos (BEHARES, 2008).

\section{Enseñanza $=$ Acto, Enseñanza $=$ Acontecimiento}

Desde la antigüedad ha sido posible distinguir dos formatos conceptuales para dar sentido a la expresión "acto de enseñanza". Uno de ellos postula que, en efecto, hay un "acto" como resultado de acciones de los participantes en la enseñanza, en tanto que actores o agentes del enseñar y del aprender. El otro formato se basa en la idea de que la enseñanza no es un acto o una acción en sentido propio, sino algo que sucede en las relaciones de los sujetos con el saber y el conocimiento, o sea un acontecimiento. $\mathrm{Si}$ se adopta el segundo formato aparecen cuestiones que son imposibles de ser representadas en términos psicológicos.

$\mathrm{El}$ acontecimiento ocurre en las dinámicas propias del saber. Su naturaleza va de "lo sabido", que implica lo representado y lo estable (o "conocimiento"), a la sujeción a lo "no sabido", que implica su carácter equívoco o en-falta, heurístico, que es el "saber" en cuanto tal. Desde la antigüedad el formato-acontecimiento ha exigido el condicionamiento dialéctico del sujeto como efecto de esas relaciones, ya que es sujeto de su conocimiento pero también de su desconocimiento, lo que nos aleja diametralmente de la discusión moderna psicológica egoica y adaptacionista, y entabla un diálogo con las corrientes teóricas que bordean la episteme del sujeto como algo que no está dado de antemano.

El siglo XX se abrió con los modelos de pensamiento propios del positivismo y del pragmatismo, que definen una realidad armónica de producción (la sociedad) y un sujeto pragmático (asegurado por una psicología) que funciona armónicamente 
en ella. También generó los antídotos a esa construcción; así, por ejemplo, en 1965 Althusser llamaba la atención sobre la inseguridad radical de la obra humana como determinación volitiva por parte de los individuos: "contrariamente a todas las apariencias todavía reinantes, no es a la psicología [...] a la que debemos estos conocimientos perturbadores sino a algunos hombres: Marx, Nietzsche y Freud" (ALTHUSSER, 2010, p. 20).

En su comentario a esta referencia en 1988, Pecheux (2006, p. 45) sostenía que el efecto subversivo de la trilogía Marx-Freud-Saussure fue un desafío intelectual que puso en entredicho la evidencia de suponer un orden humano exclusivamente en el eje psico-bio-social. Hay en ambos autores una sospecha absolutamente explícita sobre los registros de lo psicológico, que incluyen el "ego", la "consciencia", el "comportamiento" y el "sujeto epistémico", entre otros constructos semejantes. Según Pechêux, el antídoto al postulado individualista positivista-pragmatista que hace posible la psicología se encuentra en el hecho estructural propio al orden humano que denomina, siguiendo la terminología freudiano-lacaniana, "castración simbólica" (PÊCHEUX, 2006, p. 46) $)^{6}$.

Como se ha destacado ampliamente, los cuatro autores que Althusser y Pêcheux mencionaron, y otros que podrían incluirse en esa mención, produjeron teorías que pusieron en duda el postulado de un ego patrocinante, funcional a la sociedad organizada en términos de accionares positivos y armónicos. Por esta expresión queremos indicar una construcción conceptual e ideológica, el ego, que patrocina toda una visión de lo social y lo humano, en todos sus componentes (el pensamiento, la voluntad y el lenguaje incluidos), a partir de la estabilidad de los individuos, detentores de las claves necesarias a su propia justificación. En cierto sentido, podríamos también hablar de tautología egoica, que se postula desde un si-mismo evidente y positivo.

El determinismo histórico de la teoría marxista enfatizó el carácter determinado del hombre, tanto por las estructuras de clase como por el aparato ideológico. La caracterización saussureana de la Langue como un orden propio, fuera del alcance de las decisiones particulares en la acción de los individuos, estableció un recurso fuerte para definir al sujeto como un efecto de relaciones lingüísticas que son anteriores a su existencia. La teoría construida por Freud a partir del psicoanálisis dio lugar a una teoría del sujeto ya no "pura consciencia", sino dividido por su dimensión inconsciente. Estos modos de presentar las cosas del hombre, mal o bien caracterizados como "estructuralistas", con sus vaivenes y contradicciones, se sobrepuso para restar jerarquía a las psicologías egoicas.

La teoría de la enseñanza tuvo, en ese marco de referencia, la posibilidad de recuperar discusiones que habían sido postergadas por el psicologismo y el pragmatismo didacticista. Es verdad que como campo de saber en el concierto de las ciencias humanas la enseñanza demoró más que otros en recibir los influjos de estas formas críticas de pensar, pero lo ha venido haciendo desde los años de 1960. Recuperar algunas de las discusiones que habían sido postergadas como "escolásticas" o "arcaizantes2, o simplemente descartadas debido a que este ejercicio crítico no era de recibo en los ámbitos en que institucionalmente podían llegar a darse (usualmente en los 
proyectos normalistas o de los profesionales psicólogos), le permitió reformulaciones de importancia. Éstas abarcaron, centralmente, la reconceptualización del sujeto de la enseñanza en su relación con lo que habría que entender por conocimiento y por saber, ampliando las bases de lo epistémico y reduciendo la tendencia a situar las cuestiones en el plano psicológico.

\section{Comentarios finales}

Hemos intentado mostrar en este trabajo las condiciones de producción del traslado de la atención investigativa y operativa desde las figuras de la enseñanza a las del aprendizaje. La enseñanza es el campo en el que se articula la conservación del conocimiento históricamente constituido, en su encuentro con las dinámicas de la inestabilidad y la contingencia que le son constitutivas, y que llamamos saber. Por su parte, el aprendizaje es la respuesta dada por los individuos en sus procesos adaptativos y de construcción de sus herramientas intelectuales. La proclamación de la centralidad de los procesos del aprendizaje durante el siglo XX en el campo pedagógico es una transformación teórica e ideológica capital.

Esta transformación se da en el marco de procesos más amplios que vivió occidente y que tienen implicaciones mayores. Si algo nos permite jerarquizar el problema, que en sus términos supera a las discusiones técnicas y didácticas, es la promoción de la figura del ego patrocinante. Esta nueva figura está en la base de las incipientes tradiciones académicas y de implementación en el campo de la enseñanza, pero está también implicada en las políticas liberales y neoliberales inherentes a las sociedades capitalistas en materia de ciencia, tecnología y organización social, para lo cual tienen como uno de sus instrumentos principales las políticas sectoriales de educación y de enseñanza.

Asistimos en nuestra época a sus contradicciones e inconsistencias, que forman parte de las crisis de la modernidad tardía. De las aguas que han pasado nos quedan abiertas una larga serie de cuestiones, que son nuestros actuales barros. Éstos podrían reducirse a una preocupación pedagógica más, pero también podrían determinar el registro de indagaciones investigativas más proficuas.

\section{Referências}

ALLPORT, G. W. The Person in Psychology. Boston: Beacon Press. 1968.

ALTHUSSER, L. De El Capital a la filosofía de Marx. En: ALTHUSSER, L. y E. BALIBAR, Para leer E1 Capital. Mexico: Siglo XXI Editores, pp. 18-77. 2010.

AUSUBEL, D. P. Educational Psychology: a cognitive view. New York: Holt, Rinehart, and Winston. 1968.

BEHARES, L. E. De un cuerpo que responda a la palabra: un retorno a la "teoría antigua" de la enseñanza. In: BEHARES, L. E. y R. RODRÍGUEZ GIMÉNEZ (Orgs.) Cuerpo, Lenguaje y Enseñanza. Facultad de Humanidades y Ciencias-Comisión Sectorial de Investigación Científica, Montevideo, pp. 29-46.2008.

. Ego patrocinante y políticas educativas. En BOLZAN, Doris P. V. (Org.) VI Encontro Internacional de Investigadores de Políticas Educativas. Santa Maria: UFSM-AUGM, pp. 113-122. 2014. 2014. 
BEHARES, L. E. Enseñanza y educación: la contribución de Herbart a su distinción y a sus relaciones. Didáskomai, Revista de Investigaciones sobre la Enseñanza, Montevideo, № 6, pp. 13-36. 2015.

BEHARES, L. E.; R. VOLTOLINI Psicanálise e Educação: É o ensino um ato? In: Leite, N. V. y J. G. MILÁN-RAMOS (Orgs.) EntreAto: o analítico e o poético. São Paulo: Mercado de Letras, pp. 563-579. 2011.

BERRA, F. A. Apuntes para un curso de pedagogía. Montevideo: Sociedad de Amigos de la Educación Popular - Tipografía de la Nación. 1878.

BOURDIEU, P. Ce que parler veut dire. L'économie des échanges linguistiques. Paris: Fayard. 1982.

BOWEN, J. Towards an assessment of educational theory: an historical perspective. Revue Internationale de Pédagogie, 25, pp. 303-323. 1979.

BRUNER, J. S. Towards a theory of instruction. Cambridge: Harvard University Press. 1966.

BRUNER, G. Acción, Pensamiento y Lenguaje. (Compilación de J. L. LINAZA). Madrid: Alianza Editorial. 1984.

CAMILLONI, A. R. W. de, Didáctica general y didácticas específicas. In: CAMILLONI, A. R. W. de; COLS, S.; BESABE, L.; FEENEY, S. El saber didáctico. Buenos Aires: Paidós, pp. 23-40. 2007.

CAZDEN, C. El discurso en el aula. El lenguaje de la enseñanza y el aprendizaje. Barcelona: Paidós. 1991.

CHEVALLARD, Y. La trasposición didáctica. Del saber sabio al saber enseñado. Buenos Aires: Aique (tercera edición). 1998.

COLL, C. Conocimiento psicológico y práctica educativa. Introducción a las relaciones entre psicología y educación. Barcelona: Barcanova. 1988.

COULTHARD, M. An introduction to discours analysis. Londres: Longman. 1985.

DEL RÍO, P. y ÁLVAREZ, A. La actividad como problema del desarrollo. Algunos potenciales educativos del ecofuncionalismo y la psicología histórico-cultural. Cultura y Educación, 23 (4), pp. 601-619. 2010.

FAIRCLOUGH, N. Analyzing Discourse. Londres: Longman. 2003.

FERNÁNDEZ CARABALLO, A. M. Del aprendizaje en su inquietante extrañeza. In: FERNÁNDEZ CARBALLO, A. M. (Org.) El aprendizaje en cuestión. Montevideo: ediciones de la fuga.pp. 21-42. 2014.

GUMPERZ, J. Discourse strategies. Cambridge (Mass.): Cambridge University Press. 1982.

HENRY, P. A ferramenta imperfeita. Língua, Sujeito e Discurso. Campinas: Editora da Unicamp. 1992.

HERBART, J. F. Umriss pädagogischer Vorlsungen. Göttingen: Dieterichsehen Buchhandlung. 1835.

HERBART, J. F. Allgemeine Pädagogik aus dem Zweck der Erziehung abgeleitet. In: HARTENSTEIN, G. (Ed.) Johann Friedric Herbart's Sämmtliche Werke. Leipzig: Verlag von Leopold Voss, pp. 351-472.1851.

KERBRAT-ORECCHIONI, C. Les actes de langage dans le discours. París: Nathan. 2001.

LAJONQUIÈRE, L. de, Infancia e Ilusión (Psico)Pedagógica. Escritos de psicoanálisis y educación. Nueva Visión, Buenos Aires. 1999.

PARSONS, T. The Structure of Social Action. New York: The Free Press. 1968. 
PARSONS, T.; SHILDS, E. Toward a General Theory of Action: Theoretical Foundations for the Social Sciences. Cambridge, Massachusetts: Harvard University Press. 1951.

PECHEUX, M. O Discurso. Estrutura ou Acontecimento. Campinas: Pontes. 2006.

PIAGET, J. Introduction à l'épistémologie génétique. Paris: PUF. 1950.

RICOEUR, P. Freud: una interpretación de la cultura. México: Siglo Veintiuno. 2004.

SAVIANI, D. Ensino Público e outras falas sobre a Universidade. São Paulo: Cortez Editora. 1985. dos, 2000 .

Pedagogia Histórico-Crítica, primeiras aproximações. Campinas: Autores Associa-

SCHIFFRIN, D. Interactional Sociolinguistics. In: McKAY, S. L. ; HORNBERGER, E. (Eds.) Sociolinguistics and Language Teaching. Cambridge (Massachusetts): Cambridge University Press, pp. 307-328. 1996.

SKINNER, B. F. The technology of teaching. New York: Appleton-Century-Crafts. 1968.

STUBBS, M. Discourse Analysis. The sociolinguistic analysis of natural language. Chicago: University of Chicago Press. 1983.

THORNDIKE, E. L. Educational Psychology. New York: The Science Press (Kissinger Legacy Reprints, 2008). 1903.

TOLMAN, E. C. Purposive Behavior in Animals and Men. New York: Century. 1932.

VYGOTSKY, L. S. Los métodos de investigación reflexológicos y psicológicos. In: VIGOTSKY, L. S. Obras Escogidas. Madrid: Visor, Tomo I, pp. 11-340. 1991.

Historia del desarrollo de las funciones psíquicas superiores. In: VIGOTSKY, L. S. Obras Escogidas. Madrid: Visor, Tomo III, pp. 3-37. 1995.

VUYK, R. Panorámica y crítica de la Epistemología Genética de Piaget, 1965- 1980, Tomo I, Alianza, Madrid, pp. 70-100.1984.

WERTSCH, J. V. Vygotski and the social formation of mind. Londres: Harvard University Press, 1985.

\section{Notas}

\footnotetext{
${ }^{1}$ Este trabajo fue producido en los intersticios de varias líneas de investigación del Departamento de Enseñanza y Aprendizaje (Facultad de Humanidades y Ciencias de la Educación, Universidad de la República), entre 2012 y 2015.

${ }^{2}$ Se trata, mutatis mutandis, del concepto de "transposición", en el sentido de Chevallard (1998).

${ }^{3}$ En Herbart (1835), usado varias veces desde el § 57, y en Herbart (1851) en la misma dirección. Para un análisis más desarrollado, ver Behares (2015).

${ }^{4}$ La expresión "enseñanza educativa” fue utilizada tempranamente en la tradición sudamericana, como lo demuestra su aparición explícita y con caracterización detallada en Berra (1878), autor uruguayo poco conocido y menos estudiado.

${ }^{5}$ Sobre los puntos oscuros entre una y otra de estas tradiciones propias de la didáctica y su comprensión se puede consultar Chevallard (1998, Posfacio) y Camilloni (2007).
} 
${ }^{6}$ Es también éste el argumento presentado por Ricoeur, contemporáneamente a Althusser, en su conocida alusión a la "escuela de la sospecha", integrada fundamentalmente por los tres autores que señala Althusser (Ricoeur, 2004, p. 385-388).

* Professor doutor da Universidad de la República, Montevideo, Uruguay.

\section{Correspondência}

Luis E. Behares - Facultad de Humanidades y Ciencias de la Educación. Magallanes 1577 11200, Montevideo, Uruguay.

E-mail:1beharesc@yahoo.com

Recebido em 05 de setembro de 2016

Aprovado em 04 de novembro de 2016 